\title{
On the pitfalls of PTV in lung SBRT using type-B dose engine: an analysis of PTV and worst case scenario concepts for treatment plan optimization
}

Ronnie Wing King Leung ${ }^{1+}$, Mark Ka Heng Chan ${ }^{2,3^{*}+} \mathbb{D}$, Chi-Leung Chiang ${ }^{4}$, Matthew Wong ${ }^{1}$ and Oliver Blanck ${ }^{3}$

\begin{abstract}
Background: PTV concept is presumed to introduce excessive and inconsistent GTV dose in lung stereotactic body radiotherapy (SBRT). That GTV median dose prescription $\left(D_{50}\right)$ and robust optimization are viable PTV-free solution (ICRU 91 report) to harmonize the GTV dose was investigated by comparisons with PTV-based SBRT plans.

Methods: Thirteen SBRT plans were optimized for 54 Gy / 3 fractions and prescribed (i) to 95\% of the PTV ( $\left.D_{95}\right)$

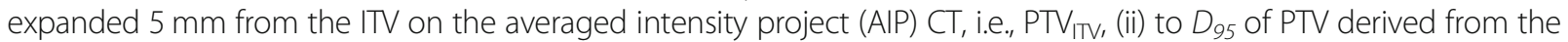
van Herk (VH)'s margin recipe on the mid-ventilation (MidV)-CT, i.e., PTV $V_{V H}$, (iii) to ITV $D_{98}$ by worst case scenario (WCS) optimization on AIP,i.e., WCS ITV and (iv) to GTV $D_{98}$ by WCS using all 4DCT images, i.e., WCS GTV. These plans were subsequently recalculated on all 4DCT images and deformably summed on the MidV-CT. The dose differences between these plans were compared for the GTV and selected normal organs by the Friedman tests while the variability was compared by the Levene's tests. The phase-to-phase changes of GTV dose through the respiration were assessed as an indirect measure of the possible increase of photon fluence owing to the type-B dose engine. Finally, all plans were renormalized to GTV $D_{50}$ and all the dosimetric analyses were repeated to assess the relative influences of the SBRT planning concept and prescription method on the variability of target dose.

(Continued on next page)
\end{abstract}

\footnotetext{
* Correspondence: Mark.Chan@uk-essen.de

${ }^{\dagger}$ Ronnie Wing Kin Leung and Mark Ka Heng Chan are Co-first authors with equal contribution.

${ }^{2}$ Department of Radiotherapy, West German Cancer Center, University

Hospital Essen, University of Duisburg-Essen, Hufelandstraße 55, 45147 Essen, Germany

${ }^{3}$ Department of Radiotherapy, University Hospital Essen, Kiel Campus, 24105 Kiel, Germany

Full list of author information is available at the end of the article
}

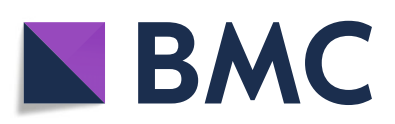

(- The Author(s). 2020 Open Access This article is licensed under a Creative Commons Attribution 4.0 International License, which permits use, sharing, adaptation, distribution and reproduction in any medium or format, as long as you give appropriate credit to the original author(s) and the source, provide a link to the Creative Commons licence, and indicate if changes were made. The images or other third party material in this article are included in the article's Creative Commons licence, unless indicated otherwise in a credit line to the material. If material is not included in the article's Creative Commons licence and your intended use is not permitted by statutory regulation or exceeds the permitted use, you will need to obtain permission directly from the copyright holder. To view a copy of this licence, visit http://creativecommons.org/licenses/by/4.0/. The Creative Commons Public Domain Dedication waiver (http://creativecommons.org/publicdomain/zero/1.0/) applies to the data made available in this article, unless otherwise stated in a credit line to the data. 
(Continued from previous page)

Results: By coverage prescriptions (i) to (iv), significantly smaller chest wall volume receiving $\geq 30$ Gy $\left(C_{V 30}\right)$ and

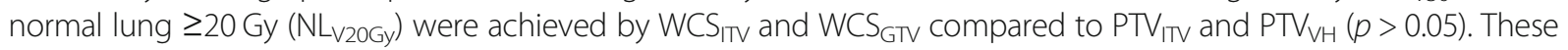
plans differed significantly in the recalculated and summed GTV $D_{2}, D_{50}$ and $D_{98}(p<0.05)$. The inter-patient variability of all GTV dose parameters is however equal between these plans (Levene's tests; $p>0.05$ ). Renormalizing these plans to GTV $D_{50}$ reduces their differences in GTV $D_{2}$, and $D_{98}$ to insignificant level $(p>0.05)$ and their inter-patient variability of all GTV dose parameters. None of these plans showed significant differences in $G T V D_{2}, D_{50}$ and $D_{98}$ between respiratory phases, nor their inter-phase variability is significant.

Conclusion: Inconsistent GTV dose is not unique to PTV concept but occurs to other PTV-free concept in lung SBRT. GTV $D_{50}$ renormalization effectively harmonizes the target dose among patients and SBRT concepts of geometric uncertainty management.

\section{Introduction}

Stereotactic body radiotherapy (SBRT) for non-small cell lung carcinomas (NSCLC) is typically delivered in free breathing condition. To limit the negative impact of respiration-induced organ motion and setup errors on its clinical benefits, passive motion management is often pursued, using either the internal target volume (ITV) concept or the mid-ventilation (MidV) concept [1]. Alternatively, passive motion management can also be realized by direct incorporation of the tumor motion into the four-dimensional (4D) optimization framework [2].

Regardless of the motion management techniques and setup uncertainty, dose optimization and prescription are invariably performed with respect to the planning target volume (PTV) to ensure, for instances, 95 and 99\% PTV coverage by 100 and $90 \%$ of the prescription dose (i.e., PTV $D_{95}=100 \%$ and $D_{99}=90 \%$ ). As suggested by Lebredonchel et al. [3], when type-B and Monte Carlo (MC) dose algorithms that model lateral electronic equilibrium (LED) are directly used to optimize to PTV $D_{95}$ a high flux of photon fluence would have to be deposited in the low density lung tissue surrounding the gross tumor volume (GTV). As a consequence, increase of dose in the lung may occur. Worse still, the GTV dose may experience increased variability only during treatment delivery as the tumor moves in and out of the high photon fluence zone over the breathing cycles. As a workaround Lacornerie et al. [4] proposed to use typeA algorithm to optimize a homogeneous fluence for which the dose distribution is ultimately calculated and renormalized to the desired prescription level using the more accurate type-B / MC algorithms. In fact, most of the major treatment planning systems (TPS) adapts type-A dose engines to increase the speed of inverse optimization for intensity-modulated (IMRT) or volumetric modulated-arc radiotherapy (VMAT). Type-B dose engine is only used at certain intermediate steps as a background dose, the so-called intermediate dose, during subsequent optimization to minimize the impact of the dose prediction and optimization convergence errors [5-7].

The latest published International Commission on Radiation Units and Measurements (ICRU) report 91 [8] continues to recommend treatment dose prescription based on PTV coverage (ICRU 91 coverage prescription) while acknowledging the increased variability of the internal GTV dose for lung SBRT using an advanced dose calculation engine. Potential solutions to improve the consistency in the reported dose and hence treatment outcomes were discussed in the report using the GTV median dose $D_{50}$ prescription and robust optimization (RO) but no further guidelines were provided. Following up the ICRU report 91 recommendations eight ACROP (Advisory Committee on Radiation Oncology) contributing centers have recently reported the variation of their prescription practices, which led to large inter-institutional and for four centers even large intra-institutional variations of the GTV/ITV doses [9]. The ACROP further made five additional clarifications, one of them recommending a minimum GTV biological equivalent mean dose of $150 \mathrm{~Gy}$. Another preliminary study from one ACROP center also demonstrated superior inter-patient variability by prescription/renormalization to ITV $D_{50}$ to prescriptions by PTV and ITV $D_{98}$ [10]. However, their results did not concern geometric uncertainty of the GTV. Current studies supporting the GTV median and mean dose optimization and prescription were mostly based on real-time tumor tracking SBRT where tumor motion was largely constrained. More importantly, very few clinical outcomes have ever been published $[11,12]$. The impact of respiration motion on the variability of target dose is still unknown for the GTV $D_{50}$ prescription/renormalization methods.

Unlike for proton therapy where $\mathrm{RO}$ has been in routine clinical practice [13], the clinical role of $\mathrm{RO}$ in photon therapy remains relatively undefined and exploratory. Since RO was introduced to the commercial TPS, there have been a few studies of its clinical application to lung SBRT but mainly focusing on the dosimetric benefits and validating the degree of robustness in reality [14]. For two 
example patient cases, Zhang et al. [15] showed that combining robust optimization with ITV - based prescription by $D_{95}$ resulted in indistinguishable dose volume histograms (DHV) of the ITV obtained on multiple breathing instances for a typical tumor motion of $1 \mathrm{~cm}$. In another phantom study, Archibald-Heeren et al. modeled the tumor motion displacement as independent scenarios and performed RO for the worst case scenario (WCS) [16]. They similarly found relatively stable tumor doses for displacement up to $2 \mathrm{~cm}$ by optimizing and prescribing to GTV $D_{99}$. However, the potential of RO to overcome the limitations of PTV has never been explored for the median dose $D_{50}$ prescription.

In the specific context of respiration-induced GTV displacement, the present study aims to validate the hypotheses

1. that using type-B dose engine with the PTV concept for dose optimization and prescription introduces significant variability of target dose and

2. that RO (by the worst case method in this study)based planning is a viable alternative to the PTV concept in lung SBRT and,

3. that prescription by GTV median dose $\left(D_{50}\right)$ can minimize the inter-patient and inter-technique variability of the reported GTV dose.

For the first argument to be valid, we hypothesized that the GTV received significantly variable doses between breathing phases. Two PTV-based optimization adapting the ITV and MidV concepts were tested using the ICRU 91 coverage prescription method. To validate the second argument, we repeated the assessment of the first argument for two WCS-based robustness optimization (hereafter called WCS optimization).The first approach is identical to Liang et al. [14] that used the ITV concept for motion encompassing. The second approach deployed all 4DCT images as independent breathing scenarios for robustness optimization. Furthermore, the dosimetric robustness was assessed by comparing the relative number of incidences that a certain target and OAR dose limit was violated in different respiratory phases. For argument 1 and 2, the inter-patient variability of GTV dose resulting from the PTV- and WCS-optimized plans were also compared. To test the third argument, all PTV and WCS-optimized plans that were prescribed by coverage according the ICRU 91 recommendation were renormalized to the GTV median dose $D_{50}$ and the above analyses were repeated.

Findings from this study are expected to provide important insight into the combination of SBRT planning concept and prescription method that produces the optimal dosimetric quality and robustness in target and organ dose during treatment, which will subsequently improve the consistency in dose reporting and multicenter clinical outcome assessment.

\section{Methods and materials}

\section{Patient selection and pre-treatment preparation}

Fourteen consecutive patients with peripherally-located lung tumors who previously received SBRT were selected for this retrospective planning study.

Helical four-dimensional computed tomography (4DCT) scan of each patient was acquired in $2 \mathrm{~mm}$ axial slices and binned into ten datasets according to respiratory phase. Using all the phase- binned 4DCT datasets an average intensity projection (AIP) image dataset was also generated on the RayStation (RaySearch Laboratories, Stockholm, Sweden; version 8a) treatment planning system (TPS).

\section{Definition of target and normal organs}

The GTV was firstly defined on one of the 4DCT dataset that was closest to the mid-ventilation (MidV) phase $\left(\mathrm{GTV}_{\text {MidV }}\right)$ [17]. It was then transformed to all other phases according to the deformation vector fields (DVFs) derived from the anatomically constrained deformation algorithm ANACONDA [18]. Finally, these GTVs from different phases were rigidly transferred onto the AIP images to produce the internal target volume (ITV). The above process also applied to the definition of normal organs.

\section{Treatment planning strategies to motion encompassing PTV-based optimization}

Two margin-based approaches were studied by optimizing to (i) the PTV expanded uniformly by $5 \mathrm{~mm}$ from the ITV, denoted as PTV ITV, on the AIP images, and (ii) the PTV expanded from the $\mathrm{GTV}_{\text {MidV }}$ by $2.5 \sum_{\text {setup }}+\beta$ $\sqrt{\sigma_{\text {setup }}{ }^{2}+\sigma_{\text {motion }^{2}}+{\sigma_{p}}^{2}}-\beta \sigma_{p}$ using the van Herk $(\mathrm{VH})$ 's margin recipe [19], denoted as $\mathrm{PTV}_{\mathrm{VH}}$, on the MidV CT. $\Sigma_{\text {setup }}$ and $\sigma_{\text {setup }}$ are the residual systemic and random errors in the tumor position including patient motion and tumor baseline drift after the online 4D cone beam computed tomography (4DCBCT) setup correction (i.e., intrafractional positioning error), $\beta=0.52$ (at a mean prescription isodose line $\sim 70 \%$ ) and $\sigma_{p}=6.4$, respectively. The motion amplitude of individual tumor is modeled as $\sigma_{\text {motion }}=1 / 3$ amplitude [19]. The GTV displacement due to respiration was implicitly accounted for by the ITV and the MidV PTV concepts, respectively.

Two partial volumetric-modulated arcs treatment (VMAT) were created using the rayArc optimization algorithm. The rayArc optimization process uses a type-A pencil-beam dose engine. For all VMAT optimization, a type-B collapsed cone convolutionsuperposition (CCCS) dose engine was introduced at the 15th iteration to calculate intermediate dose as a background dose for subsequent optimization. At the end of the VMAT optimization a final dose was calculated by CCCS. Each time further VMAT optimization 
was pursued the final dose was taken to have the same effect as an intermediate dose. The final optimized dose was prescribed to PTV $D_{95}$ at $65-75 \%$ isodose line in all cases. A total dose of $54 \mathrm{~Gy}$ for three fractions was prescribed in all cases. Dose-volume histogram (DVH) limits to different OARs were referenced from the Radiation Therapy Oncology Group (RTOG) 0236 trial [20] and the German Society of Radiation Oncology (DEGRO) guidelines [21].

\section{Robustness optimization}

In RayStation, PTV-free planning can be realized by robustness optimization ( $\mathrm{RO}$ ) based on the composite worst case method [22]. The setup uncertainty is discretized into a set of scenarios whose actual number $\left(n_{s}\right)$ depends on the size of the error. Together with nominal scenario corresponding to the planning $\mathrm{CT}$ with no assumed error, the DVH objectives are optimized for the worst case scenario (WCS) in which a robust function attaints its highest value. It is important to note that $\mathrm{RO}$ in RayStation does not treat the systematic and the random errors separately. Following ref. [23], the WCS can be approximated only for the systematic error, with the random error approximated as an additional systematic contribution. Based on the same $\Sigma_{\text {setup }}$ with $\sigma_{\text {setup }}$ as in the PTV recipe, the final WCS parameters were $3.4 \mathrm{~mm}$ (left-right), $5.3 \mathrm{~mm}$ (cranio-caudal) and $5.1 \mathrm{~mm}$ (anteroposterior) $\mathrm{mm}$. The remaining organ motion of individual patients was accounted for in two ways:

iii) implicitly by the concept of ITV in a static geometry on the AIP image.

iv) explicitly by the WCS method in a dynamic geometry that was realized by utilizing the 4DCT images of all breathing phases.

In the second WCS approach, each image set of the 4DCT composes one scenario where all the setup scenarios are examined. The total number of scenarios to be considered in the WCS optimization is then $n_{s} \cdot n_{i}$, where $n_{i}$ is the number of $4 \mathrm{DCT}$ image sets. At each iteration, minimax optimization was applied to one of all scenarios that attains the highest cost of the robust object function, i.e., the WCS. The resultant optimized plan will be robust against not just setup error but also breathing-induced tumor motion and deformation in all ten 4DCT images, hence completely margin-less. In this study, robustness was imposed to all DVH objectives of the target and the OARs.

The same two partial VMAT arcs as applied in the PTV-based planning were optimized to the ITV and the GTV to achieve $99 \%$ prescription dose coverage $\left(D_{99}\right)$ in the first and the second WCS approaches, denoted as (iii) $\mathrm{WCS}_{\mathrm{ITV}}$ and (iv) $\mathrm{WCS}_{\mathrm{GTV}}$, respectively. The same VMAT optimization process as in the PTV-optimization was adapted regarding the dose engines for optimization and prescription.

\section{Comparative analysis of PTV and WCS optimizations in static geometry}

Firstly we assessed the naïve plan optimized according to (i) to (iv) without explicit simulation of the geometric tumor displacement due to respiration. The MidV-CT was used as the common frame where all the dosimetric metrics, including mean, near-minimum and near-maximum dose in the GTV (GTV $D_{50}, D_{98}$ and $D_{2}$ ), relative volume of chest wall $(\mathrm{CW})$ receiving $30 \mathrm{~Gy}\left(\mathrm{CW}_{\mathrm{V} 30}\right)$, relative volume of normal lung (NL) receiving $20 \mathrm{~Gy}$ $\left(\mathrm{NL}_{\mathrm{V} 20}\right)$ and $5 \mathrm{~Gy}\left(\mathrm{NL}_{\mathrm{V} 5}\right)$ and the mean normal lung dose (MLD) were obtained. For this, all PTV ITV $_{\text {and }}$ WCS $_{\text {ITV }}$ plans that based their optimizations on the AIP images were recalculated on the MidV-CT.

Doses to GTV and OARs of the PTV and WCS optimized plans were compared for their difference by Friedman tests and their variance by Levene's tests using the Matlab statistics toolbox v.2019b (Mathwork Inc. MA, USA). In cases where the Friedman's tests return statistical significant at $p$-value $<0.05$, post-hoc multiple comparison tests were performed with adjusted $p$-values by Bonferroni's correction.

\section{Dosimetric variation / robustness of PTV and WCS optimizations in breathing geometry Analysis by individual respiratory phases}

If type-B dose engine does induce excessive fluence in the low density PTV border one would expect the dose received by the GTV to be higher in other breathing phases than in the planning phase. As validation, all PTV and WCS-optimized plans were firstly recalculated on every image set of the 4DCT. The resulting doses to the GTV and organs-at-risk (OARs) in individual breathing phases were statistically compared for their difference by Friedman tests and their variance by Levene's tests separately for the PTV and WCS-optimized plans. The plan robustness was defined in this context by the relative count of instances where the doses to the GTV and OARs deviate from their respective tolerance limits.

\section{Analysis over all respiratory phases}

Following the line of argument, if the PTV concept using type-B dose engine introduces excessive photon fluence the GTV would eventually accumulate significant higher dose from multiple displaced positions in the respiration cycle. As the ultimate validation, the calculated doses in individual 4DCT phase images were summed deformably according to DVFs back onto the reference MidVCT for every plan. Such deformably accumulated dose is unequivocally referred to as summed dose throughout 
the text. Similar to the evaluation in the static geometry, the summed doses to GTV and OARs were compared among all PTV and WCS-optimized plans on the reference MidV-CT for their differences by Friedman tests and their variances by Levene's tests.

The overall plan robustness was defined in this context as the dosimetric changes due to the motion effect from static to dynamic geometry and was assessed separately for different PTV and WCS WCS-optimized plans by the Wilcoxon's signed-ranked test.

\section{Dosimetric implication of prescription by GTV median dose $D_{50}$}

According to ICRU 91 report and other follow-up studies $[1,3,16]$, GTV $D_{50}$ prescription was further explored for its potential in mitigating the variability of GTV dose under the circumstances of GTV displacement by respiration. For this, all final PTV and WCS-optimized plans were renormalized so that GTV $D_{50}$ equals $54 \mathrm{~Gy}$ on respective primary planning CT images. Dosimetric and statistical analyses were then repeated as described above.

\section{Results}

PTV- and WCS-based SBRT using ICRU 91 recommended coverage prescription

\section{Dosimetric analysis in static geometry}

In the condition where no tumor displacement is concerned, all PTV and WCS-optimized plans achieved the dose constraints following the RTOG 0236 and guidelines on the reference mid-ventilation images, except for $\mathrm{CW}$. In general, WCS-optimized plans produced lower doses than PTV-optimized plans not just in the OARS but also in the GTV, as summarized in Table 1. Figure 1 shows the DVH of the GTV obtained on the MidV-CT for individual patients.
On individual patient basis, $\mathrm{CW}_{\mathrm{V} 30}$ was not met in 3 cases by $\mathrm{PTV}_{\text {ITV }}$ and 1 case by $\mathrm{PTV}_{\mathrm{VH}}$ and $\mathrm{WCS}_{\text {ITV }}$ while it was met in all cases by $\mathrm{WCS}_{\mathrm{GTV}}$. For $\mathrm{NL}_{\mathrm{V} 20}$, PTV $_{\text {ITV }}$ resulted in 6 minor deviations (within 10-15\%) and $\mathrm{PTV}_{\mathrm{VH}}$ showed 5 minor deviations according to the RTOG 0236 dose constraint. By contrast, there were 4 and 2 minor deviations resulted from WCS $_{\text {ITV }}$ and $\mathrm{WCS}_{\mathrm{GTV}}$ plans, respectively. The separation between PTV- and WCS-based SBRT plans is more pronounced in $\mathrm{NL}_{\mathrm{V} 20}$ and between $\mathrm{PTV}_{\mathrm{ITV}}, \mathrm{PTV}_{\mathrm{VH}}$ and $\mathrm{WCS}_{\mathrm{GTV}}$. We found that $\mathrm{WCS}_{\mathrm{GTV}}$ is able to reduce $\mathrm{NL}_{\mathrm{V} 5}$, on average, by $6.1 \%, \mathrm{NL}_{\mathrm{V} 20}$ by $17.9 \%$ and MLD by $12.5 \%$ comparing to $\mathrm{PTV}_{\text {ITV }}$.

Figure 1 shows the inter-patient variability of the GTV doses. Variances of each dose metrics between all PTV-based and WCS-based SBRT plans were statistically tested (Levene's tests) and were found significant for neither the GTV $\left(D_{98}, D_{50}\right.$ and $D_{2}$; all $\left.p>0.05\right)$ nor the OARs $\left(\mathrm{CW}_{\mathrm{V} 30}, \mathrm{NL}_{\mathrm{V} 5}\right.$ and $\left.\mathrm{NL}_{\mathrm{V} 20}\right)$.

\section{Dosimetric analysis by individual breathing phases}

Recalculating the PTV- and WCS-optimized plans on every image set of the 4DCT found GTV $D_{50} \geq 54$ Gy in all cases. PTV $\mathrm{ITV}_{\mathrm{ITV}}$ produced $D_{98}>54 \mathrm{~Gy}$ in all patients. There are one $\mathrm{PTV}_{\mathrm{VH}}$ plan in one phase, two $\mathrm{WCS}_{\mathrm{ITV}}$ plans in one phase and one $\mathrm{WCS}_{\mathrm{GTV}}$ plan in three phases showing $D_{98}<54$ Gy towards principally the end inhalation. The maximum differences of $D_{98}( \pm 1$ standard deviation; SD) between all 4DCT phases are $5.7 \% \pm$ $1.3,11.4 \% \pm 3.0,6.5 \% \pm 1.6$ and $6.1 \% \pm 1.5 \%$ for $\mathrm{PTV}_{\text {ITV }}$, $\mathrm{PTV}_{\mathrm{VH}}, \mathrm{WCS}_{\mathrm{ITV}}$, and $\mathrm{WCS}_{\mathrm{GTV}}$, and for GTV $D_{50}$ $1.6 \% \pm 0.5, \quad 3.4 \% \pm 0.8, \quad 1.5 \% \pm 0.4$ and $2.1 \% \pm 0.5 \%$, respectively.

Figure 2 shows the variations of GTV $D_{98}, D_{50}$ and $D_{2}$ for the 13 cases across ten breathing phases. Over all patients, none of the PTV and WCS-optimized plans

Table 1 Means and standard deviations of dose volume histogram (DVH) metrics in target and normal organs over 13 patients. All plans were prescribed by coverage according to ICRU 91

\begin{tabular}{|c|c|c|c|c|c|}
\hline & $\mathrm{PTV}_{\text {ITV }}{ }^{(i)}$ & $\mathrm{PTV}_{V H^{(i)}}$ & WCSC $_{\text {ITV }}$ (iii) & $W_{C S_{G T V}}^{\text {(iv) }}$ & $p$-value $<0.05$ \\
\hline \multicolumn{6}{|l|}{ GTV } \\
\hline$D_{98}(\mathrm{~Gy})$ & $60.6 \pm 1.8(56.1-63.7)$ & $59.6 \pm 1.5(57.0-62.3)$ & $57.8 \pm 1.7(54.8-59.6)$ & $56.4 \pm 1.0(55.2-58.6)$ & (i)-(iii); (i)-(iv); (ii)-(iv) \\
\hline$D_{50}(\mathrm{~Gy})$ & $65.7 \pm 2.2(59.7-68.1)$ & $63.1 \pm 2.2(60.0-66.5)$ & $63.7 \pm 2.2(59.9-67.2)$ & $60.5 \pm 2.1(56.4-64.8)$ & (i)-(iv) \\
\hline$D_{2}$ (Gy) & $69.3 \pm 2.3(65.1-76.3)$ & $65.4 \pm 3.0(61.3-70.7)$ & $67.2 \pm 3.561 .6-72.5)$ & $64.3 \pm 2.9(60.5-70.7)$ & (i)-(iv) \\
\hline \multicolumn{6}{|l|}{ Chest Wall } \\
\hline$V_{30 G y}\left(\mathrm{~cm}^{3}\right)$ & $26.4 \pm 16.5(4.0-60.9)$ & $19.3 \pm 9.9(1.0-38.1)$ & $14.1 \pm 8.7(0.4-31.4)$ & $12.4 \pm 7.7(0.0-29.5)$ & (i)-(iii); (i)-(iv); (ii)-(iii); (ii)-(iv) \\
\hline \multicolumn{6}{|l|}{ Lung } \\
\hline$V_{5 G y}\left(\mathrm{~cm}^{3}\right)$ & $18.1 \pm 5.7(3.3-14.0)$ & $18.7 \pm 5.0(3.6-13.5)$ & $18.1 \pm 5.8(3.1-13.4)$ & $17.0 \pm 5.6(2.8-13.4)$ & (i)-(iv); (ii)-(iv) \\
\hline$V_{20 G y}\left(\mathrm{~cm}^{3}\right)$ & $8.4 \pm 3.7(9.1-31.3)$ & $7.8 \pm 3.3(9.5-28.3)$ & $7.4 \pm 3.2(8.3-30.7)$ & $6.9 \pm 3.3(7.8-29.8)$ & (i)-(iii); (i)-(iv); (ii)-(iii); (ii)-(iv) \\
\hline$M L D(G y)$ & $4.8 \pm 1.7(2.2-7.9)$ & $4.7 \pm 1.5(2.3-7.2)$ & $4.6 \pm 1.6(2.1-7.1)$ & $4.2 \pm 1.5(1.9-7.1)$ & (i)-(iv); (ii)-(iv) \\
\hline
\end{tabular}

Abbreviation: GTV gross tumor volume, $D_{x}(G y)$ Dose to $x \%$ of the GTV, where $x=98,50$ and 2 represent the near-minimum, median and near-maximum dose respectively, $V_{x G y}\left(\mathrm{~cm}^{3}\right)$ volume receiving at least $x \mathrm{~Gy}, M L D(G y)$ mean normal lung dose; post-hoc pairwise comparisons showing $p$-values $<0.05$ are indicated when the Freidman's tests were found statistical significant 

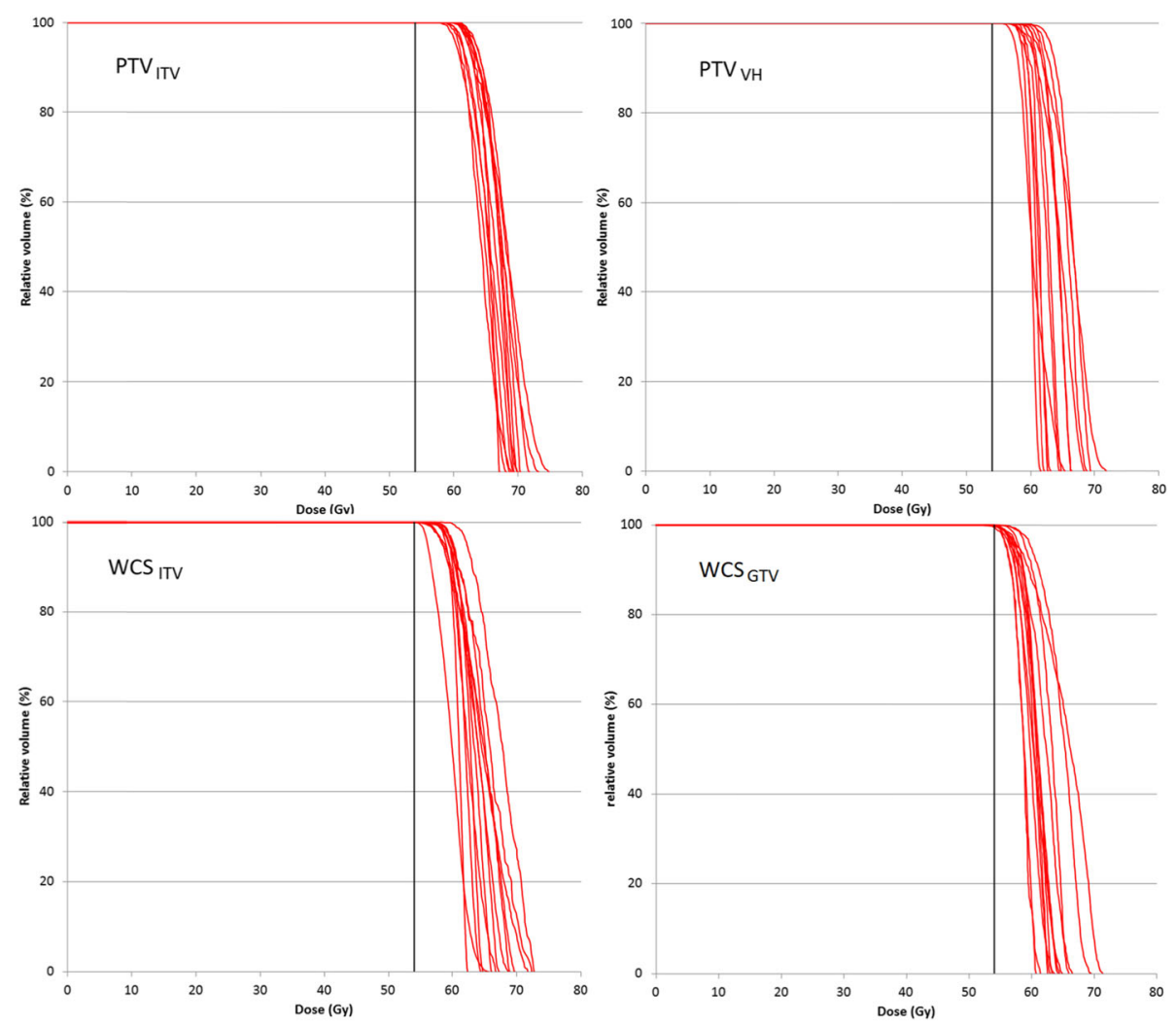

Fig. 1 Each red line represents the GTV dose volume histogram of individual patient resulting from PTV-and WCS-based SBRT plans. The black vertical line indicates the prescription dose at $54 \mathrm{~Gy}$

showed statistical importance in their differences for GTV $D_{98}$ and $D_{50}(p>0.05)$ between respiratory phases and significance was found only for $D_{2}$ with PTV $\mathrm{VH}_{\mathrm{H}}$ (Table 2). Furthermore, none of the SBRT planning concepts shows significant inter-phase variability (in terms of their variances) in GTV $D_{98}, D_{50}$ and $D_{2}(p>0.05)$.

\section{Dosimetric analysis over all breathing phases}

Table 3 shows the results of the accumulated doses obtained from the PTV and WCS- optimized plans. The accumulated GTV $D_{98}$ achieved $54 \mathrm{~Gy}$ in all plans. The changes of GTV $D_{98}, D_{50}$ and $D_{2}$ are, on average, largely limited to $1.0 \mathrm{~Gy}$. Figure 3 shows the DVH of the GTV obtained from the accumulated dose for individual patients. The inter-patient variability of doses to both the GTV and the OARs were tested to be equal among all plans (Levene's tests; all $p>0.05$ ).

For $\mathrm{CW}_{\mathrm{V} 30}$, one more patient failed the $\mathrm{CW}_{\mathrm{V} 30}$ tolerance (i.e., total 4 patients) in PTV $_{\text {ITV }}$ while one patient failed in $\mathrm{PTV}_{\mathrm{VH}}, \mathrm{WCS}_{\mathrm{ITV}}$ and $\mathrm{WCS}_{\mathrm{GTV}}$. For $\mathrm{NL}_{\mathrm{V} 20}$, one minor deviation became major deviations in $\mathrm{PTV}_{\mathrm{ITV}}$, one less minor deviations (total 4 cases) in $\mathrm{PTV}_{\mathrm{VH}}$ and the same number of minor deviations in the WCS group were found after dose summation over the tumor's excursion along the breathing cycle.

\section{PTV- and WCS-based SBRT by GTV median dose renormalization}

Dosimetric analysis in static geometry

Figure 4 shows the DVH family of the GTV after renormalized to the GTV $D_{50}$. Table 4 shows the resulting doses after renormalization to GTV $D_{50}$. Insignificant differences were found for all dose metrics, except GTV $D_{50}$ and $\mathrm{CW}_{\mathrm{V} 30}(p<0.05)$ among PTV and WCS-optimized plans. Furthermore, equal variances were found for all GTV dose metrics between optimization scenarios, except for GTV $D_{50}$.

\section{Dosimetric analysis by individual breathing phases}

Figure 5 shows the phase-to-phase variations of GTV $D_{98}, D_{50}$ and $D_{2}$ after renormalizing the summed GTV $D_{50}$. Concerning the near-minimum dose $D_{98}$, the maximum difference $( \pm 1 \mathrm{SD})$ between all $4 \mathrm{DCT}$ phases amounts to $3.3 \% \pm 0.7,6.6 \% \pm 2.0 \%, .4 .1 \% \pm 0.8$ and $5.1 \% \pm 1.2 \%$ for $\mathrm{PTV}_{\mathrm{ITV}}, \mathrm{PTV}_{\mathrm{VH}}$, WCS $\mathrm{WTV}_{\mathrm{ITV}}$, and $\mathrm{WCS}_{\mathrm{GTV}}$, and for GTV $D_{50} 2.2 \% \pm 0.6 \%, 3.4 \pm 0.9 \%, 1.5 \pm 0.4$, $2.1 \% \pm 0.5 \%$, respectively.

The statistical results of the dose differences for GTV and selected OARs between phases are given in Table 5 . None of the PTV and WCS-based SBRT concepts showed statistical significance in the difference of GTV 

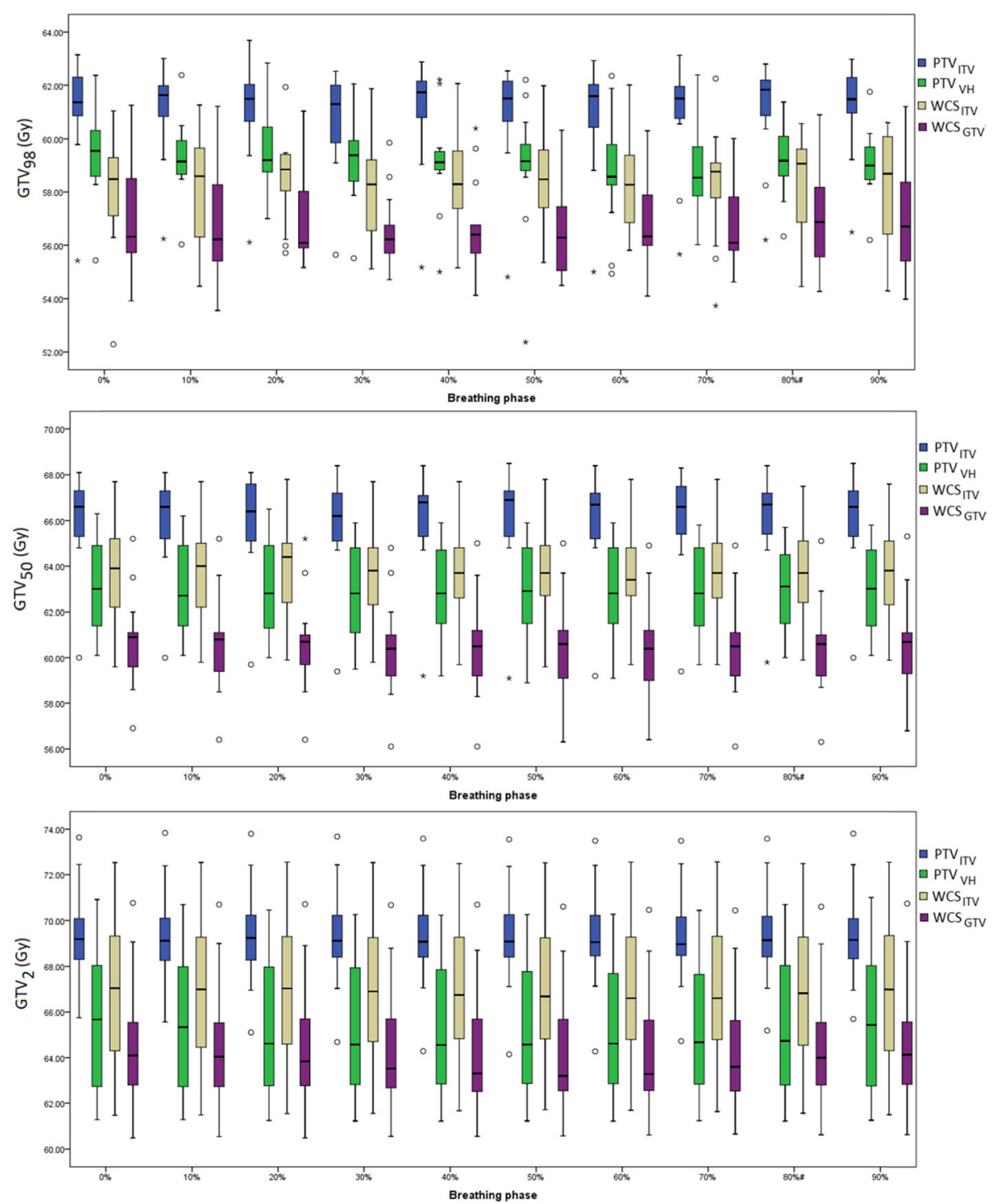

Fig. 2 Box-plots show the phase-to-phase variations of GTV $D_{98}, D_{50}$ and $D_{2}$ calculated over 13 patients for PTV and WCS-based SBRT plans. Line across the box indicates the median and the box indicate the inter-quartile range (IQR). The whiskers indicate the highest and lowest values which are no greater than 1.5 times the IQR. The outliners (opened circles) and the extremes (asterisks) are cases with values between 1.5 and 3 times, and more than 3 times the IQR, respectively

dose between phases, except for $D_{2}$ of the renormalized PTV $V_{\text {ITV }}$ plans. Furthermore, the inter-phase variability of GTV $D_{98}, D_{50}$ and $D_{2}$ was tested to be equal for all PTV and WCS-optimized plans.

\section{Dosimetric analysis over all breathing phases}

After dose accumulation, the GTV $D_{98}, D_{50}$ and $D_{2}$ and MLD change by less than $0.5 \mathrm{~Gy}$, on average. Figure 6 shows the resulting DVH of the GTV obtained for individual patients. The absolute changes of $\mathrm{CW}_{\mathrm{V} 30}$ and $\mathrm{NL}_{\mathrm{V} 5}$ by $0.6 \mathrm{~cm}^{3}$ are considered negligible despite statistical significances. Detailed results for the GTV $D_{50}$ renormalized plans are given in Table 6. Furthermore, variances of all
GTV dose metrics were tested to be equal among all renormalized PTV- and WCS-optimized plans.

\section{Discussion}

The criticism on the PTV concept in lung SBRT arises from the notion that its combination with type-B and Monte Carlo (MC) dose optimization would result in excessive and inconsistent GTV dose owing to an artificial increase of photon fluence in the low density lung tissue. Such limitation of current SBRT practice is also recognized in the recent ICRU report 91on prescribing, recording, and reporting of stereotactic treatments with small photon beams. This report further suggested that 
Table 2 Ranges of the mean and one standard deviation (SD) of the dose volume histogram metrics over10 breathing phases in13 patients. All plans were prescribed by coverage according to ICRU 91

\begin{tabular}{|c|c|c|c|c|c|c|c|c|}
\hline & PTV ITV & \multicolumn{2}{|l|}{$\mathrm{PTV}_{\mathrm{VH}}$} & \multicolumn{2}{|l|}{$\mathrm{WCS}_{\text {ITV }}$} & \multicolumn{3}{|l|}{$\mathrm{WCS}_{\mathrm{GTV}}$} \\
\hline & \multicolumn{2}{|l|}{ Range } & \multicolumn{2}{|l|}{ Range } & \multicolumn{2}{|l|}{ Range } & \multicolumn{2}{|l|}{ Range } \\
\hline & Mean ( $p$-value) & SD ( $p$-value) & Mean ( $p$-value) & SD ( $p$-value) & Mean ( $p$-value) & SD ( $p$-value) & Mean ( $p$-value) & SD ( $p$-value) \\
\hline \multicolumn{9}{|l|}{ GTV } \\
\hline$D_{98}(G y)$ & $55.7-62.5(0.65)$ & $0.3-1.0(1.00)$ & $55.5-62.2(0.32)$ & $0.2-1.8(0.91)$ & $55.0-61.5(0.96)$ & $0.3-1.1(0.99)$ & $54.8-60.5(0.90)$ & $0.2-1.2(0.70)$ \\
\hline$D_{50}(G y)$ & $59.6-68.3(0.38)$ & $0.1-0.3(1.00)$ & $60.0-65.8(0.58)$ & $0.1-0.8(1.00)$ & $59.7-67.6(0.82)$ & $0.1-0.3(1.00)$ & $56.4-65.1(0.84)$ & $0.1-0.5(1.00)$ \\
\hline$D_{2}(G y)$ & $64.9-73.6(0.32)$ & $0.0-0.6(1.00)$ & $61.2-70.5(<0.05)$ & $0.0-0.7(1.00)$ & $61.6-72.5(0.42)$ & $0.0-0.3(1.00)$ & $60.6-70.6(0.43)$ & $0.0-0.5(1.00)$ \\
\hline \multicolumn{9}{|l|}{ Chest Wall } \\
\hline$V_{30 G y}\left(\mathrm{~cm}^{3}\right)$ & $9.3-73.1(<0.01)$ & $0.7-5.3(1.00)$ & $2.8-39.9(<0.01)$ & $0.8-4.8(1.00)$ & $1.6-31.6(<0.01)$ & $0.3-4.4(1.00)$ & $1.8-29.9(<0.01)$ & $0.3-3.8(1.00)$ \\
\hline \multicolumn{9}{|l|}{ Lung } \\
\hline$V_{5 G y}\left(\mathrm{~cm}^{3}\right)$ & $9.4-29.0(0.06)$ & $0.1-1.7(0.91)$ & $9.8-25.9(<0.05)$ & $0.1-1.9(1.00)$ & $8.6-27.8(<0.05)$ & $0.1-2.0(1.00)$ & $8.0-27.0(0.09)$ & $0.1-2.0(1.00)$ \\
\hline$V_{20 G y}\left(\mathrm{~cm}^{3}\right)$ & $3.6-15.1(0.16)$ & $0.1-3.7(1.00)$ & $3.8-14.8(0.09)$ & $0.1-1.3(1.00)$ & $3.3-14.8(0.07)$ & $0.1-1.2(1.00)$ & $3.0-14.0(0.15)$ & $0.1-1.3(1.00)$ \\
\hline MLD (Gy) & $2.3-8.3(<0.01)$ & $0.0-0.6(1.00)$ & $2.4-7.9(<0.01)$ & $0.0-0.5(1.00)$ & $2.2-7.8(<0.01)$ & $0.0-0.5(1.00)$ & $2.0-7.4(<0.01)$ & $0.0-0.5(1.00)$ \\
\hline
\end{tabular}

Note: $p$ values were obtainedfrom Freidman's tests and Levene's tests comparing the differences and the variances between ten breathing phases, respectively, in 13 patients per SBRT optimization method

Abbreviations are the same as in Table 1

robust optimization and GTV-based prescriptions are viable options to possibly improve the overall dosimetric accuracy and quality while reducing the variability in dose reporting and potentially even in dose prescription.

By analyzing the dosimetric variability and robustness resulting from two common PTV-based and two other worse case-based robust optimization methods, this study is now able to provide more clarifications to the pitfalls of PTV concept in lung SBRT. Additionally, by analyzing further the dosimetric results by different dose prescription methods according to the ICRU recommended coverage prescription and GTV median dose prescription, we identified the dominant factor that contributes to the variability of GTV dose.

Table 3 Accumulated dose volume histograms (DVH) over the entire breathing cycle for PTV and WCS optimized plans based on prescription by coverage according to ICRU 91

\begin{tabular}{|c|c|c|c|c|c|c|}
\hline & & $\mathrm{PTV}_{\text {ITV }}{ }^{(i)}$ & PTV $V_{V H}^{\text {(ii) }}$ & WCSC ITV $_{\text {Tiii) }}$ & $W C S_{G T V}{ }^{(i v)}$ & \\
\hline & $\begin{array}{l}\text { Motion effect } \\
\text { (Wilcoxon's) }\end{array}$ & Mean \pm SD (range) & Mean \pm SD (range) & Mean \pm SD (range) & Mean \pm SD (range) & $\begin{array}{l}\text { (i) vs.(ii) vs.(iii) vs.(iv) } \\
p \text {-value }<0.05\end{array}$ \\
\hline \multicolumn{7}{|l|}{ GTV } \\
\hline \multirow[t]{2}{*}{$D_{98}(G y)$} & & $61.5 \pm 1.8(56.3-63.0)$ & $59.7 \pm 1.6(56.5-62.5)$ & $59.0 \pm 1.8(55.5-62.0)$ & $57.2 \pm 1.9(55.1-61.3)$ & (i)-(iii); (i)-(iv); (ii)-(iv) \\
\hline & $p$-value & 0.003 & 1.000 & 0.022 & 0.092 & \\
\hline \multirow[t]{2}{*}{$D_{50}(\mathrm{~Gy})$} & & $65.7 \pm 2.2(59.6-67.9)$ & $62.8 \pm 2.1(59.8-65.6$ & $63.2 \pm 2.2(59.7-67.2)$ & $60.3 \pm 2.2(56.1-65.0)$ & (i)-(iv) \\
\hline & $p$-value & 0.581 & 0.092 & 0.022 & 0.267 & \\
\hline \multirow[t]{2}{*}{$D_{2}(G y)$} & & $69.1 \pm 2.3(64.5-73.3)$ & $65.2 \pm 3.0(61.1-70.1)$ & $66.9 \pm 3.6(61.5-72.4)$ & $64.0 \pm 2.8(60.5-70.3)$ & (i)-(iv) \\
\hline & $p$-value & $<0.01$ & $<0.01$ & $<0.01$ & $<0.01$ & \\
\hline \multicolumn{7}{|l|}{ Chest Wall } \\
\hline \multirow[t]{2}{*}{$V_{30 G y}\left(\mathrm{~cm}^{3}\right)$} & & $28.5 \pm 18.7(6.3-72.4)$ & $20.0 \pm 10.6(1.1-41.6)$ & $16.9 \pm 9.0(1.7-31.4)$ & $13.0 \pm 8.4(1.0-32.5)$ & (i)-(iii); (i)-(iv); (ii)-(iv); (iii)-(iv); \\
\hline & $p$-value & 0.20 & 0.02 & $<0.01$ & $<0.01$ & \\
\hline \multicolumn{7}{|l|}{ Lung } \\
\hline \multirow[t]{2}{*}{$V_{5 G y}\left(\mathrm{~cm}^{3}\right)$} & & $16.5 \pm 5.8(7.7-30.0)$ & $17.3 \pm 5.1(8.0-26.7)$ & $16.8 \pm 5.7(7.5-28.7)$ & $16.1 \pm 5.6(7.2-28.3)$ & (ii)-(iv) \\
\hline & $p$-value & 0.14 & 0.17 & 0.20 & 0.03 & \\
\hline \multirow[t]{2}{*}{$V_{20 G y}\left(\mathrm{~cm}^{3}\right)$} & & $8.3 \pm 3.8(3.6-15.4)$ & $7.8 \pm 3.3(3.6-13.8)$ & $7.7 \pm 3.7(3.3-15.1)$ & $7.0 \pm 3.4(2.8-13.4)$ & (i)-(iii); (i)-(iv); (ii)-(iv); (iii)-(iv) \\
\hline & $p$-value & 0.27 & 0.11 & 0.25 & 0.10 & \\
\hline \multirow[t]{2}{*}{$M L D(G y)$} & & $4.0 \pm 1.6(1.8-7.2)$ & $4.1 \pm 1.3(1.9-6.1)$ & $3.9 \pm 1.4(1.8-6.3)$ & $3.8 \pm 1.4(1.7-6.3)$ & (i)-(iv); (ii)-(iv) \\
\hline & $p$-value & 0.06 & 0.38 & 0.22 & 0.18 & \\
\hline
\end{tabular}



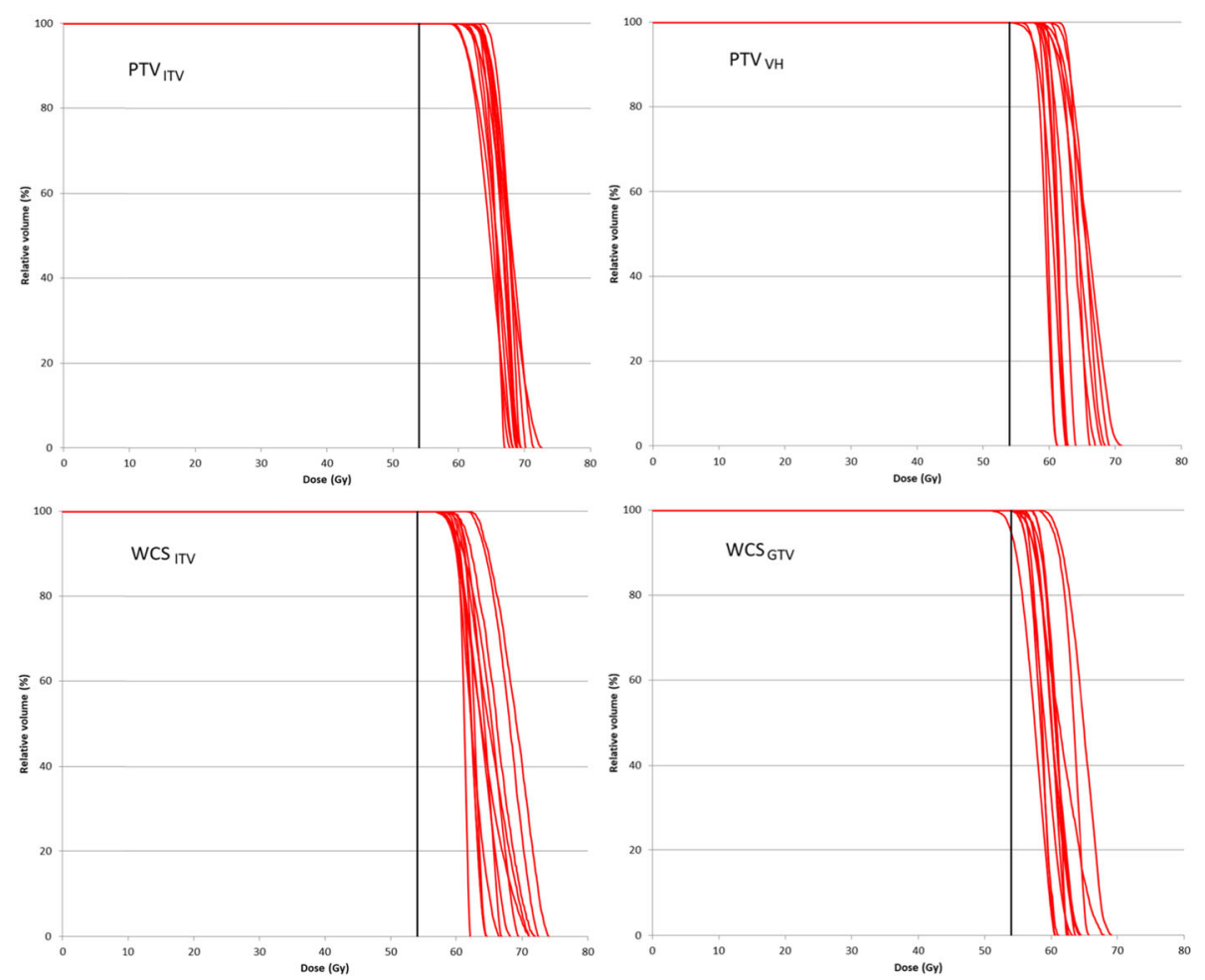

Fig. 3 Each red line represents the GTV DVH of individual patient obtained from the accumulated dose for PTV and WCS-based SBRT plans. The black vertical line indicates the prescription dose at $54 \mathrm{~Gy}$

PTV- and WCS-based SBRT using ICRU 91 recommended coverage prescription

SBRT plans optimized and prescribed to the PTV resulted in significant overexposure to the GTV compared to those plans optimized for WCS as expected. The GTV receives much higher dose, with the GTV median dose $D_{50}$ about 17 and $22 \%$ over the prescription dose for ITV-based and mid-ventilation based PTV optimizations, respectively. Although higher dose to the GTV is generally not a concern and even desired for SBRT, part of the excessive dose is in effect burdened by the surrounding normal organs including the normal lung and chest wall that are encompassed in the PTV. For lesions that are close to the chest wall, the volume receiving $\geq 30$ Gy $\left(\mathrm{V}_{30}\right)$ was reduced significantly by up to 29.5 $\mathrm{cm}^{3}(74 \%)$ and 31.4 (73\%) by using WCS optimization on the averaged intensity projection (AIP) image to the ITV and on all 4DCT images directly to the GTV in comparisons to the conventional PTV approach based on the ITV. The dosimetric benefit of WCS optimization to limit the chest wall dose was also reported by Zhang et al. [21]. In their study, 8 of 20 patient plans optimized and prescribed to PTV showed chest wall dose above the limit whereas all WCS plans optimized to the ITV fulfilled the dose constraint. In this study, we showed that WCS optimized to the GTV can further improve the chest wall dose.
Besides the dosimetric inferiority to WCS optimization, the other major pitfall of PTV concept for plan optimization is that inconsistent GTV doses between individual patients (i.e., inter-patient variability) occur even with the same PTV prescription. However, our results clearly demonstrated that inconsistent GTV dose is not unique to the PTV concept. Other methods that avoid the PTV concept in SBRT planning equally suffer from inconsistent GTV doses. Specifically, robust optimization that replaces the PTV concept by the worst case method also shows inconsistent GTV dose. This was evidenced by the equivalent variances of GTV $D_{98}, D_{50}$ and $D_{2}$ among all PTV and WCS-optimized plans (Table 1). In principle, one would expect zero or minimal variability of GTV $D_{98}$ at and close to the prescription point of GTV $D_{98}$ or ITV $D_{98}$ in the WCS-optimized plans. Recall that robust optimization in this study was implemented to ensure the prescription dose in the worst case scenario, that is, the GTV $D_{98}$ was optimized to equal to or at least $54 \mathrm{~Gy}$ in the worst case scenario but it could be any values $>54$ Gy in other scenarios. Since the nominal scenario does not necessarily coincide with the worst case scenario, and in fact hardly does, GTV $D_{98}$ does not necessarily arrive exactly at 54 Gy in the nominal scenario and hence variability. On the other hand, any renormalization made to equalize GTV $D_{98}$ to 54 Gy in the nominal scenario would 

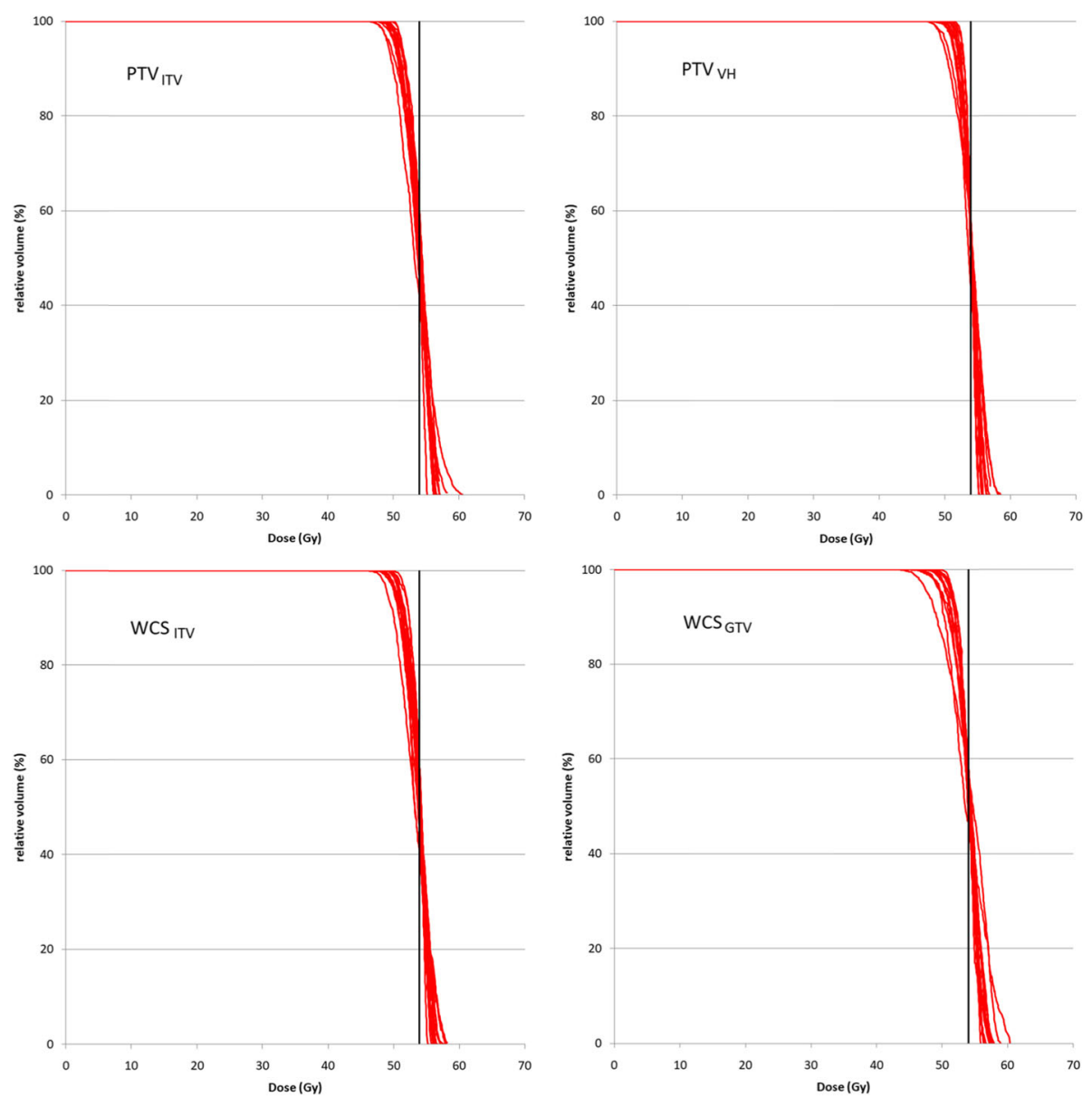

Fig. 4 Each red line represents the GTV DVH of individual patient obtained from PTV and WCS-based SBRT plans by GTV $D_{50}$ prescription. The black vertical line indicates the prescription dose at $54 \mathrm{~Gy}$

Table 4 Means and standard deviations of dose volume histogram (DVH) metrics in target and normal organs over 13 patients. All plans were renormalized based on GTV median dose $D_{50}$

\begin{tabular}{|c|c|c|c|c|c|}
\hline & $\mathrm{PTV}_{\text {ITV }}^{(i)}$ & $\mathrm{PTV}_{\mathrm{VH}}{ }^{\text {(ii) }}$ & WCSC $_{\text {ITV }}{ }^{\text {(iii) }}$ & WCS $_{\text {GTV }}{ }^{\text {(iv) }}$ & $p$-value $<0.05$ \\
\hline \multicolumn{6}{|l|}{ GTV } \\
\hline$D_{98}(G y)$ & $50.3 \pm 0.6(48.7-51.0)$ & $51.3 \pm 1.0(49.1-52.4)$ & $50.0 \pm 1.4(47.9-52.5)$ & $49.8 \pm 1.2(46.4-51)$ & (ii)-(iv) \\
\hline$D_{50}(G y)$ & $54.1 \pm 0.2(53.8-54.5)$ & $54.0 \pm 0.0(54.0-54.0)$ & $54.1 \pm 0.3(53.5-54.6)$ & $54.0 \pm 0.0(54.0-54.0)$ & \\
\hline$D_{2}(G y)$ & $56.7 \pm 0.9(55.1-58.8)$ & $56.0 \pm 0.9(55.1-57.7)$ & $57.0 \pm 1.1(55.0-58.9)$ & $56.8 \pm 0.7(55.8-58.4)$ & (ii)-(iii) \\
\hline \multicolumn{6}{|l|}{ Chest Wall } \\
\hline$V_{30 G y}\left(\mathrm{~cm}^{3}\right)$ & $8.2 \pm 7.9(0.0-24.5)$ & $8.1 \pm 6.4(0.0-18.8)$ & $6.7 \pm 4.8(0.0-15.0)$ & $6.6 \pm 4.8(0.0-15.4)$ & \\
\hline \multicolumn{6}{|l|}{ Lung } \\
\hline$V_{5 G y}\left(\mathrm{~cm}^{3}\right)$ & $17.1 \pm 5.3(2.6-13.6)$ & $17.9 \pm 4.3(2.9-11.7)$ & $17.4 \pm 5.1(2.6-11.9)$ & $16.7 \pm 5.1(2.4-12.3)$ & (ii)-(iv) \\
\hline$V_{20 G y}\left(\mathrm{~cm}^{3}\right)$ & $6.6 \pm 3.3(7.7-30.0)$ & $6.7 \pm 3.0(8.0-26.8)$ & $6.2 \pm 3.0(7.4-28.7)$ & $6.1 \pm 3.1(7.1-28.4)$ & (ii)-(iii); (ii)-(iv) \\
\hline MLD (Gy) & $3.9 \pm 1.6(1.8-7.2)$ & $4.0 \pm 1.3(1.9-6.1)$ & $3.9 \pm 1.4(1.8-6.3)$ & $3.7 \pm 1.3(1.7-6.4)$ & (ii)-(iv) \\
\hline
\end{tabular}

Abbreviations are the same as in Table 1 

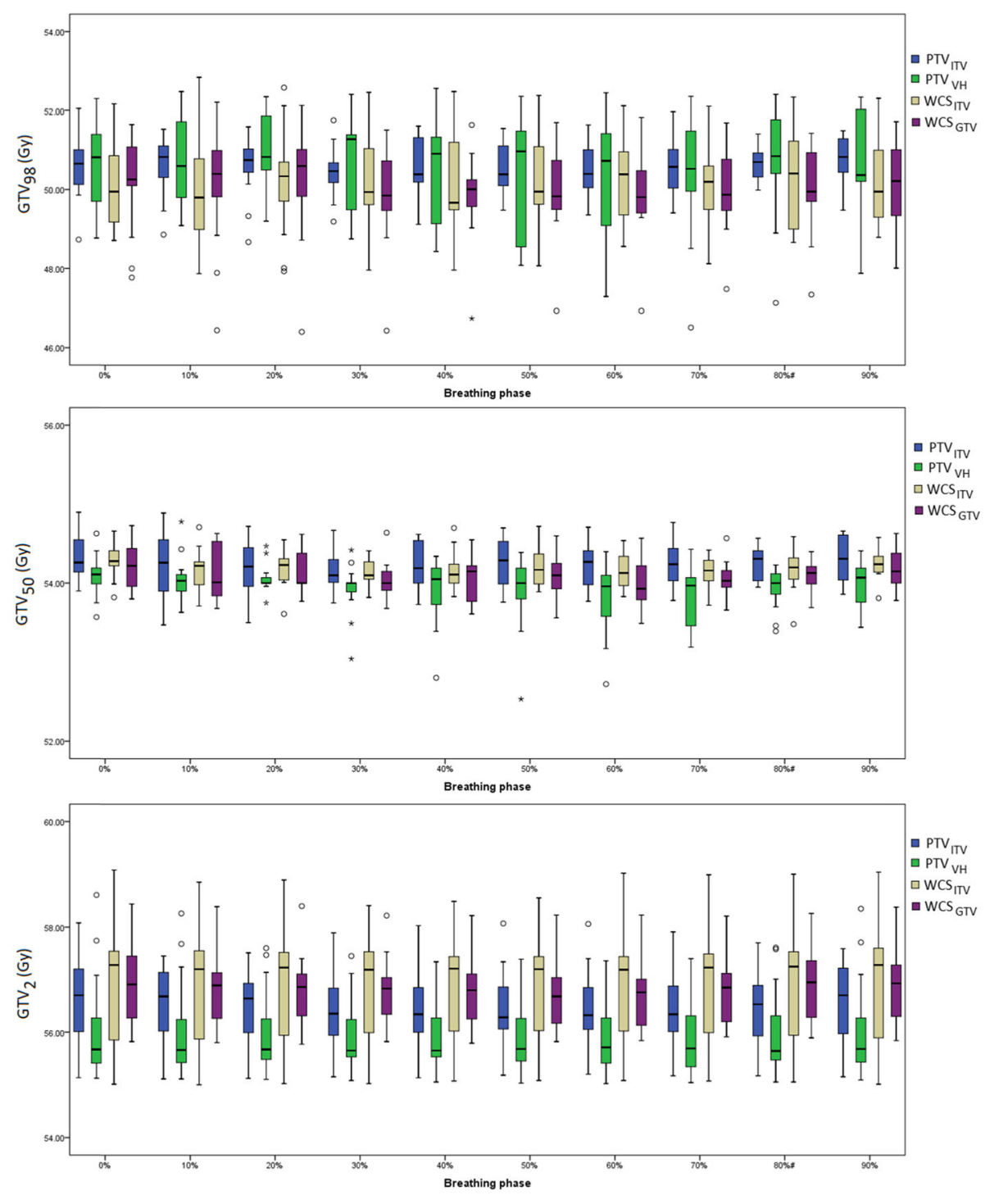

Fig. 5 Box-plots show the phase-to-phase variations of GTV $D_{98}, D_{50}$ and $D_{2}$ calculated over 13 patients for PTV and WCS-optimized plans based on GTV $D_{50}$ prescription. Values of the box, whiskers, outliners and extremes are described in Fig. 2; The same 2 Gy scale is set as in Fig. 2

invalidate the plan robustness that was achieved to ensure the prescription dose for the worst case scenario.

When Lacornerie et al. [11] initially argued against type-B dose engine for dose optimization using the PTV concept, they claimed "the GTV will be overexposed when it moves into the regions with increased photon fluence" but without providing results to assess the magnitude of the matter. Following this line of argument, if type-B dose engine did induce excessive photon fluence in the low density PTV border one would expect the dose received by the GTV to be higher in other respiratory phases than in the planning phase. We therefore followed the phaseto-phase changes in the GTV doses. Our results show that all GTV dose parameters, except for $D_{2}$ using the mid-ventilation concept, were statistically equal among the ten 4DCT images for the PTV-optimized plans. Guckenberger et al. [24] previously optimized for the PTV coverage $D_{95}$ on the end-exhale CT, in which case the type-B dose engine would in principle drive the optimizer to deposit the maximal fluence at the opposite end-inhale position. Interestingly, the authors found no significant GTV dose differences when these plans were recalculated on the end-inhale CT. Maximum differences of $6.9 \pm 3.1 \%$ and $2.4 \pm 1.8 \%$ for GTV $D_{99}$ and $D_{50}$ were reported, respectively. This study observed smaller maximum differences of $2.7 \pm 1.4 \%$ and $0.9 \pm 0.5 \%$ for GTV $D_{98}$ and $D_{50}$, respectively. The discrepancy is presumably attributed to the different planning CT datasets (end-exhale vs. AIP images) for which the fluence optimization were carried out. 
Table 5 Ranges of the mean and one standard deviation (SD) of the dose volume histogram metrics over10 breathing phases in13 patients. All plans were normalized based on GTV median dose $D_{50}$

\begin{tabular}{|c|c|c|c|c|c|c|c|c|}
\hline & \multicolumn{2}{|l|}{$\begin{array}{l}\text { PTV }{ }_{\text {ITV }} \\
\text { Range }\end{array}$} & \multicolumn{2}{|l|}{$\begin{array}{l}\text { PTV } \\
\text { Range }\end{array}$} & \multicolumn{2}{|l|}{$\begin{array}{l}\text { WCS } \\
\text { Range } \\
\end{array}$} & \multicolumn{2}{|l|}{$\begin{array}{l}W_{C S} \text { GTV } \\
\text { Range }\end{array}$} \\
\hline & Mean ( $p$-value) & SD ( $p$-value) & Mean ( $p$-value) & SD ( $p$-value) & Mean ( $p$-value) & SD ( $p$-value) & Mean ( $p$-value) & SD ( $p$-value) \\
\hline \multicolumn{9}{|l|}{ GTV } \\
\hline$D_{98}(\mathrm{~Gy})$ & $49.2-51.5(0.82)$ & $0.2-0.5(0.91)$ & $48.2-52.4(0.32)$ & $0.1-0.9(0.67)$ & $48.4-52.3(0.90)$ & $0.2-0.6(0.99)$ & $51.4-47.1(0.59)$ & $0.1-1.0(0.98)$ \\
\hline$D_{50}(G y)$ & $54.0-54.6(0.57)$ & $0.1-0.4(0.40)$ & $53.5-54.4(0.24)$ & $0.0-0.7(0.25)$ & $54.0-54.6(0.29)$ & $0.0-0.2(0.86)$ & $53.7-54.5(0.76)$ & $0.0-0.4(0.21)$ \\
\hline$D_{2}$ (Gy) & $55.2-57.8(<0.05)$ & $0.0-0.6(1.00)$ & $55.1-57.6(0.25)$ & $0.0-0.6(1.00)$ & $55.0-58.8(0.49)$ & $0.0-0.3(1.00)$ & $55.8-58.3(0.10)$ & $0.0-0.4(1.00)$ \\
\hline \multicolumn{9}{|l|}{ Chest Wall } \\
\hline$V_{30 G y}\left(\mathrm{~cm}^{3}\right)$ & $0.1-32.8(<0.01)$ & $0.0-4.4(0.99)$ & $0.1-19.1(<0.01)$ & $0.0-3.0(0.97)$ & $0.1-14.9(<0.01)$ & $0.0-2.6(0.86)$ & $0.1-16.4(<0.01)$ & $0.0-2.5(0.99)$ \\
\hline \multicolumn{9}{|l|}{ Lung } \\
\hline$V_{5 G y}\left(\mathrm{~cm}^{3}\right)$ & 7.9-28.0 (0.08) & $0.1-1.6(1.00)$ & $8.2-24.5(<0.05)$ & $0.1-1.9(1.00)$ & $7.7-26.6(<0.05)$ & $0.1-1.6(1.00)$ & $7.4-25.7(<0.03)$ & $0.1-2.0(1.00)$ \\
\hline$V_{20 G y}\left(\mathrm{~cm}^{3}\right)$ & $2.8-12.8(0.09)$ & $0.1-1.4(1.00)$ & $3.1-12.9(0.11)$ & $0.1-1.2(1.00)$ & $2.7-12.2(0.08)$ & $0.1-1.3(1.00)$ & $2.6-12.0(<0.05)$ & $0.0-1.3(1.00)$ \\
\hline MLD (Gy) & $1.8-6.9(<0.01)$ & $0.0-0.5(1.00)$ & $2.0-6.5(<0.01)$ & $0.0-0.5(1.00)$ & $1.9-6.5(<0.01)$ & $0.0-0.5(1.00)$ & $1.8-6.1(<0.01)$ & $0.0-0.5(1.00)$ \\
\hline
\end{tabular}

Note: $p$ values were obtainedfrom Freidman's tests and Levene's tests comparing the differences and the variances between ten breathing phases, respectively, in 13 patients per SBRT planning method Abbreviations are the same as in Table 1

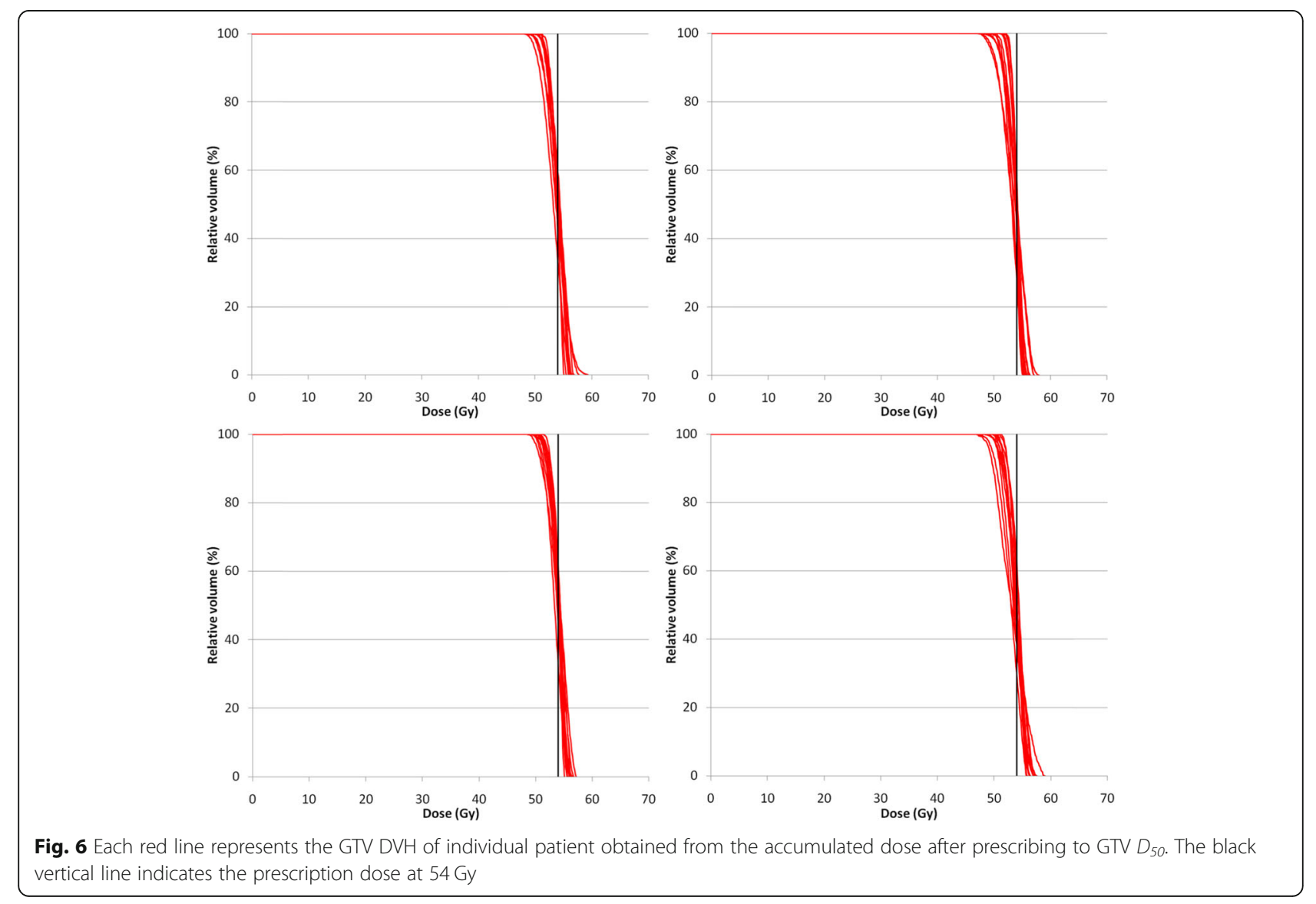


Table 6 Accumulated dose volume histograms (DVH) over the entire breathing cycle. All plans were renormalized to GTV median dose $D_{50}$

\begin{tabular}{|c|c|c|c|c|c|c|}
\hline & & $\mathrm{PTV}_{\text {ITV }}{ }^{(\mathrm{i})}$ & $\operatorname{PTV}_{\mathrm{VH}}{ }^{\text {(ii) }}$ & WCSC $_{\text {ITV }}{ }^{\text {(iii) }}$ & WCS $_{G T V}{ }^{\text {(iv) }}$ & \\
\hline & $\begin{array}{l}\text { motion effect } \\
\text { (Wilcoxon's) }\end{array}$ & Mean \pm SD (range) & Mean \pm SD (range) & Mean \pm SD (range) & Mean \pm SD (range) & $\begin{array}{l}\text { (i) vs.(ii) vs.(iii) vs.(iv) } \\
p \text {-value }<0.05\end{array}$ \\
\hline GTV & & & & & & \\
\hline$D_{98}(\mathrm{~Gy})$ & & $50.8 \pm 0.6(49.6-51.7)$ & $51.0 \pm 1.3(48.5-52.6)$ & $50.4 \pm 1.3(48.6-52.5)$ & $50.3 \pm 1.1(47.4-51.5)$ & \\
\hline & $p$-value & 0.003 & 1.000 & 0.003 & 0.92 & \\
\hline$D_{50}(\mathrm{~Gy})$ & & $54.0 \pm 0.2(53.7-54.4)$ & $53.8 \pm 0.3(53.1-54.2)$ & $54.0 \pm 0.2(53.7-54.3)$ & $53.9 \pm 0.2(53.4-54.3)$ & (ii)-(iv) \\
\hline & $p$-value & 0.267 & 0.022 & 0.092 & 0.092 & \\
\hline$D_{2}(G y)$ & & $56.4 \pm 0.7(55.0-57.7)$ & $55.8 \pm 0.8(55.0-57.4)$ & $56.7 \pm 1.0(54.9-58.7)$ & $56.5 \pm 0.7(55.6-58.0)$ & \\
\hline & $p$-value & $<0.01$ & $<0.01$ & $<0.01$ & $<0.01$ & \\
\hline Chest Wall & & & & & & \\
\hline$V_{30 G y}\left(\mathrm{~cm}^{3}\right)$ & & $8.8 \pm 8.4(0.0-27.2)$ & $8.7 \pm 6.9(0.0-21.2)$ & $7.3 \pm 5.5(0.0-17.5)$ & $7.1 \pm 5.3(0.0-17.5)$ & \\
\hline & $p$-value & 0.01 & 0.02 & 0.02 & 0.02 & \\
\hline Lung & & & & & & \\
\hline$V_{5 G y}\left(\mathrm{~cm}^{3}\right)$ & & $16.5 \pm 5.8(7.7-30.0)$ & $17.3 \pm 5.1(8.0-26.7)$ & $16.8 \pm 5.7(7.5-28.7)$ & $16.1 \pm 5.6(7.2-28.3)$ & (ii)-(iv) \\
\hline & $p$-value & 0.01 & 0.04 & 0.02 & 0.03 & \\
\hline$V_{20 G y}\left(\mathrm{~cm}^{3}\right)$ & & $6.7 \pm 3.3(2.7-13.5)$ & $6.8 \pm 3.1(2.9-12.0)$ & $6.3 \pm 3.0(2.6-11.9)$ & $6.2 \pm 3.1(2.5-12.2)$ & (ii)-(iii); (ii)-(iv) \\
\hline & $p$-value & $<0.01$ & $<0.05$ & 0.02 & $<0.01$ & \\
\hline$M L D$ (Gy) & & $4.0 \pm 1.6(1.8-7.2)$ & $4.1 \pm 1.3(1.9-6.1)$ & $3.9 \pm 1.4(1.8-6.3)$ & $3.8 \pm 1.4(1.7-6.3)$ & (ii)-(iv) \\
\hline & $p$-value & 0.01 & 0.01 & $<0.01$ & $<0.01$ & \\
\hline
\end{tabular}

Abbreviations are the same as in Table 1

Note: The motion effect is the evaluated by comparing the recalculated and accumulated dose on the MidV-CT

Here, we attempt to offer an explanation to the negligible GTV dose difference among breathing phases from the principles of conventional radiotherapy and SBRT. In conventional VMAT-based radiotherapy, a uniform dose profile (e.g., $+/-5 \%$ ) across the PTV is often demanded and achieved by a fluence profile that is typically characterized with horns at the PTV edge to compensate for the beam penumbra. Thus, the GTV may experience an increase of fluence when it moves towards the PTV border. The magnitude of this fluence horn increases from water density to lung density to counterbalance the deteriorating condition of charged particle equilibrium. By contrast, SBRT allows higher dose in the tumor center (as much as $167 \%$ when normalized to the maximum dose at $60 \%$ on the PTV surface). In this case, the "horn" effect diminishes as the demand of photon fluence is counterbalanced by the allowed lower dose to the region around the PTV edge. The other possible reason could be that commercial planning system generally switches the type-A dose engine to type-B dose engines only at certain steps for fluence correction during the dose optimization and in final dose calculation.

Additionally, we examined the variances of different GTV dose parameters among the ten respiratory phases. Our hypothesis is that if type-B dose engine did drive up the photon fluence in the PTV-optimized plans the inter-phase variability of these GTV dose parameters would become significantly different. This hypothesis is based on the fact that individual patients have different characteristics (e.g., tumor size, motion amplitude, lung density, etc) and hence the extent to which the photon fluence were to be driven up would vary substantially. When the GTV moves in different spatio-temporal positions of the respiratory cycle it would receive photon fluence of varying degree from phase to phase that is patient dependent. Nonetheless, we found that both PTV and WCS optimizations resulted in equal variances of all GTV dose parameters among the ten respiratory phases. Interestingly, the inter-quartile ranges (IQR) of GTV $D_{98}$ resulting from WCS optimized plans using all 4DCT images were found to be more variable than from other PTV-optimized plans. This large but insignificant variability of GTV $D_{98}$ is hypothesized to have originated from the specific worst case optimization method. Compared to the voxel-wise and objective-wise robust methods, the composite worst case method implemented by the RayStation planning systems behaves to maximally minimize the objective value on the worst case scenario at the cost of higher objective values and thus larger dosimetric fluctuation in many other possible scenarios [25]. Since the worst case scenario may correspond to different breathing phases with different patient characteristics, relatively large variability of $D_{98}$ among breathing phases was observed. Nonetheless, by WCS optimization, 
particularly using all 4DCT images, the highest robustness was achieved to prevent the dose limits in the normal tissues from being exceeded when the target is displaced into different respiratory positions.

As the final validation, we compared the optimized dose on a single CT and the recalculated doses summed over all 4DCT images. Such comparisons offer clarifications to two important issues concerning the nonconsistency of PTV concept in lung SBRT. Firstly, if type-B dose engine induced excessive fluence in PTVbased optimization, the GTV would eventually accumulate significant higher dose when it moved into different breathing phases. However, no clear indication of overexposure to the GTV can be associated with PTV-based optimization (Table 3). The GTV $D_{50}$ and $D_{2}$ obtained from PTV-optimized plans for the ITV and mid-ventilation concepts changed by 0.3 Gy only after dose summation and on the contrary decreased rather than increased. The significant increase of GTV $D_{98}$ in the PTV-optimized plans based on the ITV concept does not appear to be related to the type-B dose engine because it did not occur to the other PTV-optimized plans that adopted the mid-ventilation concept. Instead, it was presumably caused the systematic change in using the AIP images for dose optimization to the mid-ventilation images for dose accumulation. For the rather extreme situation using the end-exhale $\mathrm{CT}$ for fluence optimization, neither did Guckenberger et al. [24] observe serious problem of excessive build up of photon fluence at the opposite end-inhalation that caused a significant change in the overall GTV dose either. More interestingly, the authors too found an increase rather than a decrease in the summed GTV dose (presumably $D_{95}$ ) by less than $1 \%$ or 0.7 Gy only. Among all GTV dose parameters, $D_{50}$ appears to be the most robust against changes showing no statistical significance except for the ITV-based robust optimization. Based on these results, we conclude that type-B dose engine, per se, does not significantly increase the GTV dose. The significantly higher GTV dose in the PTV-optimized plans than WCS optimized plans is rather a direct consequence of the prescription method.

Secondly, equal variances of the GTV dose parameters among the PTV and WCS-optimized plans are still observed after dose summation over the ten 4DCT images. The inter-patient variability (one standard deviation) changes only by $0.1 \mathrm{~Gy}$ after dose summation in all but the GTV $D_{98}$ of the WCS-optimized plans $(0.9 \mathrm{~Gy})$. This simply means that the inconsistency of GTV dose cannot be easily resolved by migrating from the PTV concept to robust optimization irrespective of the type- $\mathrm{B}$ dose engine $[1,14]$. For the same reasoning, we would argue that using two classes of dose engines, a type-A for fluence optimization followed by a type-B for subsequent dose calculation and renormalization will not resolve the inconsistent GTV dose either. We would further argue that PTV concept, in its very design to account for geometric uncertainty, shall not be considered as a pitfall. Consistency of clinical outcome report shall not be compromised provided that the advanced dose engines are used to estimate and report the GTV dose parameters following the ESTRO ACROP recommended guidelines [11].

\section{PTV- and WCS-based SBRT by GTV median dose renormalization}

Lebredonchel et al [14] suggested that prescribing based on $50 \%$ mass of the PTV can somewhat stabilize variability of the target dose but they concluded further that moving away from the PTV concept for prescription remains the only solution if using type-B dose engine. They came to this conclusion because the GTV median dose $D_{50}$ differs substantially with variable lung density and tumor size when prescription is done to the PTV. However, this conclusion is considered as partly true only because our results already showed that other PTV-free concept by the worst case method does not stabilize the target doses either when the ICRU recommended prescription by coverage (i.e., GTV $D_{98}$ or ITV $D_{98}$ ) was followed. Instead, the prescription method has the major impact on the variability of GTV dose. After renormalization based on GTV $D_{50}$, the separations of the DVH families became much packed together for all plans optimized using different concepts (Fig. 4), as compared to those obtained from prescription by coverage (Fig. 1). The resulting SDs of $D_{98}$ and $D_{50}$ and $D_{2}$ are limited to $1 \mathrm{~Gy}$ for PTV- and 1.4 Gy for WCS-optimized plans, respectively. Focusing on the concept of ITV as motion encompassing, Lang et al. similarly showed that the SDs of PTV $D_{98}$ and $D_{50}$ and ITV $D_{98}$ of 38 patients are limited to $1.5 \mathrm{~Gy}$ after ITV $D_{50}$ renormalization to $57 \mathrm{~Gy}$ [18]. They also showed that the ITV $D_{50}$ renormalization is superior to renormalization by ITV/PTV coverage $D_{98}$ as it can reduce the variability of PTV and ITV dose parameters among delivery techniques (dynamic conformal arc vs. VMAT). More importantly, the differences of GTV $D_{98}$ and $D_{50}$ and $D_{2}$ among optimized plans based on the PTV concept and the WCS method (Table 4) were found to reduce markedly. These results are still valid despite the variation of tumor position in the respiration cycle, with GTV $D_{50}$ being the only dose parameter that showed statistically significant difference. However, the absolute difference of $0.2 \mathrm{~Gy}$ is deemed clinically unimportant. Same as the results of coverage prescription, the median dose turned out to be the most robust against uncertainty of tumor position among other GTV dose parameters.

The effect of GTV $D_{50}$ renormalization is also marked at the phase to phase level (Fig. 5). The median of all GTV dose parameters became much closer among the plans 
that adopted different concepts for setup and motion compensation. Compared to the prescription by coverage method recommended by ICRU 91 report, the maximum inter-phase difference of GTV $D_{98}$ was reduced by 2.4 , 4.8 and $2.4 \%$ and $1.0 \%$ for PTV optimization by the ITV and mid-ventilation concepts, and WCS optimization to the ITV and GTV, respectively.

In summary, when SBRT plans are directly prescribed or renormalized to the GTV median dose $D_{50}$

1. the consistency of GTV dose across the nearminimum, median, and near-maximum points is significantly improved, i.e. reduced inter-patient variability

2. harmonization of GTV dose is made possible for lung SBRT plans that adopt different concepts to handle geometric uncertainty caused by respiratory motion.

The first point simply implies that one can continue with the PTV concept for dose planning. The second point implies that consistent GTV dose shall be ensured between SBRT centers employing either the PTV concept or the worst case scenario concept in dose planning, and different delivery techniques as indicated by Lang et al. [18].

On the other hand, one may question the value of robust optimization concerning its computational overheads, if by $D_{50}$ GTV prescription alone can simply harmonize the GTV dose among optimization solutions. From the normal tissue dose perspective, our phase-by-phase analysis indicates that WCS optimization in general improved the dosimetric robustness, resulting in the fewest number of dose deviations from the OAR limits. Furthermore, lower $\mathrm{NL}_{\mathrm{V} 5}$ and MLD (Table 3) during respiration were constantly observed in the WCS optimization group regardless of the prescription method. In particular, WCS optimization to the GTV using all 4DCT images resulted in the lowest normal tissue dose and highest robustness against deviation of normal tissue dose limit among all optimization methods.

\section{Limitation of the study}

This study was designed by assuming the same amount of geometric uncertainties from tumor motion and patient setup in the calculation of the PTV and in the definition of the WCS parameters. Nonetheless, our results considered exclusively the uncertainty of tumor position due to breathing motion. The validity of our results shall hold because uncertainty of respiratory motion, which is considered as systematic in our phase-to-phase analysis for the GTV dose changes, is much greater than that of setup limited to millimeter accuracy with stereotactic image guidance.

The other limitation is the small number of patients which may subject our results to bias. Only 2 out of 13 patients showed tumor motion more than $1 \mathrm{~cm}$. It is unclear whether our dosimetric results will remain unchanged if more patients with larger amplitude of tumor motion are included.

We also acknowledge that the exact formulation of the robustness optimization may have an influence on the dosimetric results [22]. Despite the numerous robustness optimization algorithms, there is only one commercial planning system that makes robust optimization available for clinical use. This study, like many other previous ones, was based on the worst case scenario optimization from the same planning system. Lastly, this study focused on a certain type (convolution-superposition) and class (type-B) of dose engine. Systematic difference between Monte Carlo and type B dose engines is well known especially in cases where extreme electron charged disequilibrium exists [26]. Further investigation with Monte Carlo dose engine is warranted to generalize the present findings.

\section{Conclusions}

The pitfalls of PTV concept have no association with type-B dose engine in lung SBRT. Inconsistent target dose is not unique to the PTV concept but the worst case method implemented in the robust optimization. Prescription by coverage, regardless to the PTV $D_{95}$ or GTV $D_{98}$ in common practice has the major impact on the consistency of GTV dose. GTV median dose prescription or renormalization can effectively decrease the inter-patient and inter-optimization method (PTV and worst case scenario) variability of GTV dose.

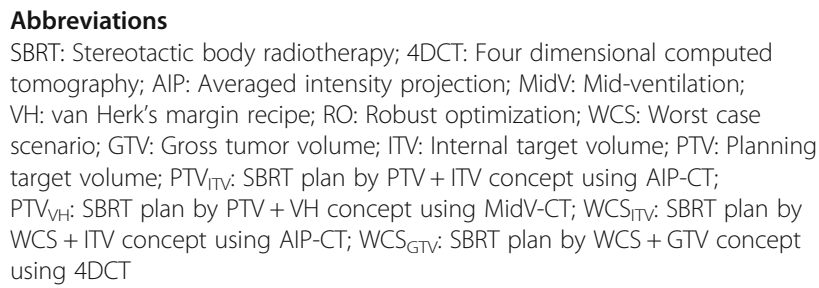

\section{Acknowledgements}

We acknowledge support by the Open Access Publication Fund of the University of Duisburg-Essen.

\section{Authors' contributions}

$\mathrm{RL}$ and MKHC conceived the study. RL and MW performed the treatment planning and the collection of dosimetric data for analysis. MKHC and OB performed the data analysis and prepared the manuscript. $\mathrm{CL}$ reviewed and proof-read the manuscript. The author(s) read and approved the final manuscript.

\section{Funding}

Not applicable.

\section{Availability of data and materials}

The datasets related to patient information are not available. Other data used and / or analyzed during the present study are available from the corresponding author on reasonable request. 


\section{Ethics approval and consent to participate}

This study was approved by an institutional review board (study reference: NTWC/CREC/17100).

\section{Consent for publication}

Not applicable.

\section{Competing interests}

The authors declare no conflicts of interests.

\section{Author details}

'Department of Clinical Oncology, TuenMun Hospital, Hong Kong, SAR, China. ${ }^{2}$ Department of Radiotherapy, West German Cancer Center, University Hospital Essen, University of Duisburg-Essen, Hufelandstraße 55, 45147 Essen, Germany. ${ }^{3}$ Department of Radiotherapy, University Hospital Essen, Kiel Campus, 24105 Kiel, Germany. ${ }^{4}$ Department of Clinical Oncology, The University of Hong Kong, Hong Kong, China.

Received: 20 March 2020 Accepted: 17 May 2020

Published online: 29 May 2020

\section{References}

1. Guckenberger M, Andratschke N, Dieckmann K, Hoogeman MS, Hoyer M, Hurkmans C, Tanadini-Lang S, Lartigau E, Méndez Romero A, Senan S, Verellen D. ESTRO ACROP consensus guideline on implementation and practice of stereotactic body radiotherapy for peripherally located early stage non-small cell lung cancer. Radiother Oncol. 2017;124:11-7.

2. Nohadani O, Seco J, Bortfeld T. Motion management with phase-adapted 4D-optimization. Phys Med Biol. 2010:55:5189-202.

3. Lebredonchel S, Lacornerie T, Rault E, Wagner A, Reynaert N, Crop F. About the non-consistency of PTV-based prescription in lung. Phys Med. 2017;44: $177-87$

4. Lacornerie T, Lisbona A, Mirabel X, Lartigau E, Reynaert N. GTV-based prescription in SBRT for lung lesions using advanced dose calculation algorithms. Radiat Oncol. 2014;9:223.

5. Li Y, Rodrigues A, Li T, Yuan L, Yin F-F, Wu QJ. Impact of dose calculation accuracy during optimization on lung IMRT plan quality. J Appl Clin Med Phys. 2015;16:219-28.

6. Bzdusek K, Friberger H, Eriksson K, Hårdemark B, Robinson D, Kaus M. Development and evaluation of an efficient approach to volumetric arc therapy planning. Med Phys. 2009;36:2328-39.

7. Bokrantz R. Multicriteria optimization for volumetric-modulated arc therapy by decomposition into a fluence-based relaxation and a segment weightbased restriction. Med Phys. 2012;39:6712-25.

8. Seuntjens J, Lartigau EF, Cora S, Ding GX, Goetsch S, Nuyttens J, Roberge D. ICRU report 91. Prescribing, recording, and reporting of stereotactic treatments with small photon beams. J ICRU. 2014:4(2):1-160.

9. de Jong EEC, Guckenberger M, Andratschke N, Dieckmann K, Hoogeman MS, Milder M, Møller DS, Nyeng TB, Tanadini-Lang S, Lartigau E, et al. Variation in current prescription practice of stereotactic body radiotherapy for peripherally located early stage non-small cell lung cancer: recommendations for prescribing and recording according to the ACROP guideline and ICRU report 91. Radiother Oncol. 2019;142:217-23 .

10. Tanadini-Lang S, Wilke L, Karava K, Andratschke N, Ehrbar S, Guckenberger M. Dose normalization in lung SBRT based on ICRU 91 and comparison to alterative normalization methods. Radiother Oncol. 2018;127:S1213-4.

11. Baumann R, Chan MKH, Pyschny F, Stera S, Malzkuhn B, Wurster S, Huttenlocher S, Szücs M, Imhoff D, Keller C, et al. Clinical results of mean GTV dose optimized robotic-guided stereotactic body radiation therapy for lung tumors. Front Oncol. 2018:8:171.

12. Bibault J-E, Mirabel X, Lacornerie T, Tresch E, Reynaert N, Lartigau E. Adapted prescription dose for Monte Carlo algorithm in lung SBRT: clinical outcome on 205 patients. PLoS One. 2015;10:e0133617.

13. Liu W, Schild SE, Chang JY, Liao Z, Chang Y-H, Wen Z, Shen J, Stoker JB, Ding $X, H u Y$, et al. Exploratory study of $4 D$ versus $3 D$ robust optimization in intensity modulated proton therapy for lung cancer. Int J Radiat Oncol Biol Phys. 2016;95:523-33.

14. Liang X, Zheng D, Mamalui-Hunter M, Flampouri S, Hoppe BS, Mendenhall N, Li Z. ITV-based robust optimization for VMAT planning of stereotactic body radiation therapy of lung cancer. Pract Radiat Oncol. 2019;9:38-48.
15. Zhang X, Rong Y, Morrill S, Fang J, Narayanasamy G, Galhardo E, Maraboyina S. Croft C, xia F, Penagaricano J: robust optimization in lung treatment plans accounting for geometric uncertainty. J Appl Clin Med Phys. 2018;19:19-26.

16. Archibald-Heeren BR, Byrne MV, Hu Y, Cai M, Wang Y. Robust optimization of VMAT for lung cancer: Dosimetric implications of motion compensation techniques. J Appl Clin Med Phys. 2017;18:104-16.

17. Wolthaus JWH, Schneider C, Sonke J-J, van Herk M, Belderbos JSA, Rossi MMG, Lebesque JV, Damen EMF. Mid-ventilation CT scan construction from four-dimensional respiration-correlated CT scans for radiotherapy planning of lung cancer patients. Int J Radiat Oncol Biol Phys. 2006;65:1560-71.

18. Weistrand O, Svensson S. The ANACONDA algorithm for deformable image registration in radiotherapy. Med Phys. 2015;42:40-53.

19. Wolthaus JWH, Sonke J-J, van Herk M, Belderbos JSA, Rossi MMG, Lebesque $J V$, Damen EMF. Comparison of different strategies to use four-dimensional computed tomography in treatment planning for lung cancer patients. Int Radiat Oncol Biol Phys. 2008;70:1229-38.

20. Xiao Y, Papiez L, Paulus R, Timmerman R, Straube WL, Bosch WR, Michalski J Galvin JM. Dosimetric evaluation of heterogeneity corrections for RTOG 0236: stereotactic body radiotherapy of inoperable stage I-II non-small-cell lung cancer. Int J Radiat Oncol Biol Phys. 2009;73:1235-42.

21. Guckenberger $M$, Andratschke $N$, Alheit $H$, Holy R, Moustakis $C$, Nestle $U$, Sauer O. Deutschen Gesellschaft für R: definition of stereotactic body radiotherapy: principles and practice for the treatment of stage I non-small cell lung cancer. Strahlenther Onkol. 2014;190:26-33.

22. Fredriksson A. A characterization of robust radiation therapy treatment planning methods - from expected value to worst case optimization. Med Phys. 2012;39:5169-81.

23. Korevaar EW, Habraken SJM, Scandurra D, Kierkels RGJ, Unipan M, Eenink MGC, Steenbakkers RJHM, Peeters SG, Zindler JD, Hoogeman M, Langendijk JA. Practical robustness evaluation in radiotherapy - a photon and protonproof alternative to PTV-based plan evaluation. Radiother Oncol. 2019;141: 267-74.

24. Guckenberger M, Wilbert J, Krieger T, Richter A, Baier K, Meyer J, Flentje M. Four-dimensional treatment planning for stereotactic body radiotherapy. Int J Radiat Oncol Biol Phys. 2007;69:276-85.

25. Fredriksson A, Bokrantz R. A critical evaluation of worst case optimization methods for robust intensity-modulated proton therapy planning. Med Phys. 2014;41:081701.

26. Li J, Galvin J, Harrison A, Timmerman R, Yu Y, Xiao Y. Dosimetric verification using monte carlo calculations for tissue heterogeneity-corrected conformal treatment plans following RTOG 0813 dosimetric criteria for lung cancer stereotactic body radiotherapy. Int J Radiat Oncol Biol Phys. 2012;84:508-13.

\section{Publisher's Note}

Springer Nature remains neutral with regard to jurisdictional claims in published maps and institutional affiliations.

Ready to submit your research? Choose BMC and benefit from:

- fast, convenient online submission

- thorough peer review by experienced researchers in your field

- rapid publication on acceptance

- support for research data, including large and complex data types

- gold Open Access which fosters wider collaboration and increased citations

- maximum visibility for your research: over $100 \mathrm{M}$ website views per year

At BMC, research is always in progress.

Learn more biomedcentral.com/submissions 